\title{
Quantitative DNA methylation analyses reveal stage dependent DNA methylation and association to clinico-pathological factors in breast tumors
}

Jovana Klajic ${ }^{1,2}$, Thomas Fleischer ${ }^{2,3}$, Emelyne Dejeux ${ }^{4}$, Hege Edvardsen ${ }^{3}$, Fredrik Warnberg ${ }^{5}$, Ida Bukholm²,6, Per Eystein Lønning ${ }^{7}$, Hiroko Solvang ${ }^{3}$, Anne-Lise Børresen-Dale ${ }^{2,3}$, Jörg Tost ${ }^{4}$ and Vessela N Kristensen ${ }^{1,2,3^{*}}$

\begin{abstract}
Background: Aberrant DNA methylation of regulatory genes has frequently been found in human breast cancers and correlated to clinical outcome. In the present study we investigate stage specific changes in the DNA methylation patterns in order to identify valuable markers to understand how these changes affect breast cancer progression.

Methods: Quantitative DNA methylation analyses of 12 candidate genes ABCB1, BRCCA1, CDKN2A, ESR1, GSTP1, IGF2, MGMT, HMLH1, PPP2R2B, PTEN, RASSF1A and FOXC1 was performed by pyrosequencing a series of 238 breast cancer tissue samples from DCIS to invasive tumors stage I to IV.

Results: Significant differences in methylation levels between the DCIS and invasive stage II tumors were observed for six genes RASSF1A, CDKN2A, MGMT, ABCB1, GSTP1 and FOXC1. RASSF1A, ABCB1 and GSTP1 showed significantly higher methylation levels in late stage compared to the early stage breast carcinoma. Z-score analysis revealed significantly lower methylation levels in DCIS and stage I tumors compared with stage II, III and IV tumors. Methylation levels of PTEN, PPP2R2B, FOXC1, ABCB1 and BRCA1 were lower in tumors harboring TP53 mutations then in tumors with wild type TP53. Z-score analysis showed that TP53 mutated tumors had significantly lower overall methylation levels compared to tumors with wild type TP53. Methylation levels of RASSF1A, PPP2R2B, GSTP1 and FOXC1 were higher in ER positive vs. ER negative tumors and methylation levels of PTEN and CDKN2A were higher in HER2 positive vs. HER2 negative tumors. Z-score analysis also showed that HER2 positive tumors had significantly higher z-scores of methylation compared to the HER2 negative tumors. Univariate survival analysis identifies methylation status of PPP2R2B as significant predictor of overall survival and breast cancer specific survival.
\end{abstract}

Conclusions: In the present study we report that the level of aberrant DNA methylation is higher in late stage compared with early stage of invasive breast cancers and DCIS for genes mentioned above.

Keywords: Breast cancer, DNA methylation, Methylation index, Stage, TP53

\footnotetext{
* Correspondence: v.n.kristensen@medisin.uio.no

'Department of Clinical Molecular Biology and Laboratory Science (EpiGen),

Akershus University hospital, Division of Medicine, 1476 Lørenskog, Norway

${ }^{2}$ Institute for Clinical Medicine, Faculty of Medicine, University of Oslo, 0372

Oslo, Norway

Full list of author information is available at the end of the article
} 


\section{Background}

Breast cancer is the most common form of malignant disease in women worldwide and also the principal cause of death from cancer among women globally. In Norwegian women breast cancer accounts for approximately $23 \%$ of all cancers and $\sim 800$ women die as a result of the disease every year. Breast cancer is a heterogeneous disease with distinct histopathological, genetic and epigenetic characteristics. Epigenetic regulation is critical for normal growth and development and provides a layer of transcriptional control. Epigenetic alterations which occur in transformed cells involve changes in DNA methylation including global hypomethylation and focal hypermethylation, histone modifications and nucleosomal remodeling [1]. Epigenetic changes are considered to be an early event in tumor development and one of the hallmarks of cancer [2]. The degree of DNA methylation in the promoter region of tumor suppressor genes, transcription factors and drug response genes may play a role in the initiation of cancer, tumor progression and response to treatment. Identification of early epigenetic changes in breast cancer might give valuable markers for early detection and contribute to the understanding of how these changes affect the progression of the disease and prognosis for the patient. We previously described treatment-specific DNA methylation patterns in $432 \mathrm{CpGs}$ in the promoter regions of 14 genes in samples from 75 patients with locally advanced breast cancer treated with doxorubicin [3]. Further, we identified four novel genes ( $A B C B 1, F O X C 1, P P P 2 R 2 B$ and PTEN) that were found to be already aberrantly methylated in DCIS (Ductal carcinoma in situ), a pre-invasive stage of breast cancer [4] and that were also found to be methylated in the locally advanced breast cancers [3]. These findings raised the question if DNA methylation patterns evolve between the different stages of breast cancers and pre-neoplastic lesions or are similar and independent of tumor stage. In the present study, using the same candidate gene approach as previously described [3,4], we performed a large-scale analysis of 12 candidate genes and determined quantitatively the DNA methylation patterns of the 12 genes in 238 breast cancer patients of all stages from early premalignant DCIS to advanced metastatic disease. In addition to the analysis of stage dependent DNA methylation patterns, associations between additional clinico-pathological factors of breast cancer such as grade and ER status and DNA methylation patterns of these 12 genes were assessed.

\section{Methods}

\section{Patient material}

A total of 238 samples were included in present study: 75 patients with locally advanced breast cancer that were enrolled in a prospective study evaluating predictive factors for response to doxorubicin [3] and 35 patients treated with 5-FU and Mitomycin for locally advanced breast cancer [5], patients in both cohorts were admitted to the Haukeland University Hospital in Norway between 1991 and 2001; 57 samples from a series of 212 breast cancers samples collected from Ullevål University Hospital (Norway) between 1990 and 1994 [6]; 71 tumors from a populationbased cohort including 854 women diagnosed between 1986 and 2004 in Uppsala, Sweden with either: a) pure ductal carcinoma in situ of the breast (DCIS), b) an invasive breast cancer, $15 \mathrm{~mm}$ or less, without an in situ component or c) a mixed lesion, i.e., a lesion with both an invasiveand an in situ component [4]. Clinical and molecular characteristics of the tumors are given in Table 1. DNA from six normal breast tissues was included to identify the DNA methylation baseline in normal tissues. Normal breast tissue was obtained from women who underwent a biopsy of the mammary gland because of mammographic screening and for whom histology confirmed the presence of only normal tissue. All patients had given informed consent, and the project was approved by the local ethical committee.

\section{Methylation assays}

DNA concentrations were determined using the Quant-i ${ }^{\mathrm{TM}}$ dsDNA broad range assay kit (Invitrogen, Cergy Pontoise, France) and normalized to a concentration of $50 \mathrm{ng} / \mu \mathrm{l}$. One $\mu \mathrm{g}$ of DNA was bisulphite converted using the MethylEasy $^{\mathrm{Tm}}$ HT Kit for Centrifuge (Human Genetic Signatures, North Ryde, Australia) according to the manufacturer's instructions. Quantitative DNA methylation analysis of the bisulphite treated DNA was performed by pyrosequencing or - in case of several sequencing primers - by serial pyrosequencing [7]. Oligonucleotides for PCR amplification and pyrosequencing (Additional file 1) were synthesized by Biotez (Buch, Germany) [3]. In the present study, same candidate gene approach was used as previously described $[3,4]$ with the difference in number of covered CpGs (205 in our case) because of absence of variability. These genes were initially selected on the following basis: previous reports of DNA methylation in breast tumors or at least breast cancer cell lines $(A B C B 1$ [8], BRCA1 [9], CDKN2A [9], ESR1 [10], GSTP1 [11], IGF2 [12], MGMT [9], MLH1 [9], PPP2R2B [13], PTEN [14], RASSF1A [15]) and genes displaying variation in breast cancer gene expression profiles (FOXC1 [16]).

\section{Statistical analysis}

The average value of methylation for all CpGs in a target region was calculated for each sample and each gene. Although there was some stochastic variation between different $\mathrm{CpG}$ positions, the overall methylation level was quite constant and $\mathrm{CpG}$ positions were highly correlated in the analyzed regions. A sample was considered 1) 
Table 1 Clinical characteristics of the analyzed samples

Clinicopatho

T0

$\mathrm{T} 1$

$\mathrm{T} 2$

T3

T4

Unknown

Stage

DCIS

I

II

III

IV

Unknown

Lymphnode status

No

N1

N2

Unknown

Grade

G1

G2

G3

Unknown

HER2 status

Negative

Positive

Unknown

Progesteron receptor

Negative

Positive

Unknown

Estrogene receptor

Negative

Positive

Unknown

TP53 mutation

Wild type

Mutated

Unknown

Distant metastasis

No distant metastasis

Distant metastasis

Unknown
$\mathrm{N}(\%)$

26(11\%)

67(28.3\%)

35(14.8\%)

64(27\%)

45(18.9\%)

1

26(11.5\%)

65(28.9\%)

20(8.9\%)

92(40.9\%)

22(9.8\%)

13

114(50.7\%)

76(34.9\%)

32(14.4\%)

16

42(17.7\%)

128(54\%)

$67(28.3 \%)$

1

60(57.1\%)

45(42.9\%)

133

69(29.2\%)

167(70.8\%)

2

53(22.7\%)

$180(77.3 \%)$

5

174(73.4\%)

63(26.6\%)

1

214(90.3\%)

23(9.7\%)

1
Table 1 Clinical characteristics of the analyzed samples (Continued)

\begin{tabular}{ll}
\hline Molecular subtypes & \\
Luminal A & $52(30.8 \%)$ \\
Luminal B & $34(20.1 \%)$ \\
ERRB2 & $29(17.2 \%)$ \\
Basal & $37(21.9 \%)$ \\
Normal & $17(10 \%)$ \\
Unknown & 69 \\
\hline
\end{tabular}

Unknown data was excluded from total when the percentage was calculated.

hypermethylated if the percentage of DNA methylation was higher than the sum of two times the standard deviation and mean of the normal samples, 2) normal-like methylation if the\% DNA methylation was in range of two times the standard deviation $+/-$ mean of the normal sample and 3) hypomethylated if\% DNA methylation was lower than two times standard deviation - mean of normal sample. The aggregated quantitative DNA methylation data is presented in Table 2. Differences in the presence of methylation were determined by a twosided Fisher test (for variables with two categories) and Chi- squared tests for variables with three or more categories. Odds ratio and 95\% confidence intervals were calculated for two-categorical variables. Differences in the distribution of methylation were assessed by the non-parametric Mann-Whitney test (on parameters with 2 categories) or the Kruskal-Wallis test analysis on parameters with more than two categories. All obtained p-values were corrected with the Bonferroni correction method in which the p-values are multiplied by the number of comparisons. The methylation index of samples (Z-score) was calculated as: (methylation level of each sample - mean

Table 2 Quantitative methylation data

\begin{tabular}{lrrrr}
\hline Gene & $\begin{array}{r}\text { Mean of } \\
\text { samples }\end{array}$ & $\begin{array}{r}\text { SD of } \\
\text { samples }\end{array}$ & $\begin{array}{r}\text { Mean of } \\
\text { normal }\end{array}$ & $\begin{array}{r}\text { SD of } \\
\text { normal }\end{array}$ \\
\hline BRCA1 & 85.36 & 11.32 & 85.48 & 6.15 \\
RASSF1A & 30.92 & 17.34 & 3.37 & 0.99 \\
PTEN & 5.15 & 4.07 & 2.83 & 1.28 \\
PPP2R2B & 10.83 & 9.80 & 3.18 & 1.17 \\
CDKN2A & 4.68 & 4.64 & 3.58 & 1.08 \\
MLH1 & 2.94 & 3.85 & 2.72 & 0.47 \\
MGMT & 4.21 & 3.53 & 4.48 & 1.11 \\
ABCB1 & 16.18 & 17.25 & 2.63 & 1.23 \\
IGF2 & 38.28 & 9.13 & 40.7 & 2.41 \\
GSTP1 & 17.18 & 17.27 & 3.73 & 1.01 \\
FOXC1 & 13.41 & 13.23 & 3.6 & 1.98 \\
ESR1 & 2.92 & 2.50 & 4.43 & 2.94 \\
\hline & & & &
\end{tabular}


Table 3 Methylation status of 12 genes in normal tissue, DCIS and invasive breast cancer patients

\begin{tabular}{|c|c|c|c|c|c|c|c|c|c|c|c|c|c|}
\hline \multirow{3}{*}{ GENE } & \multirow[b]{3}{*}{ Methylation status } & \multicolumn{3}{|c|}{ Normal } & \multirow{3}{*}{$\begin{array}{r}\text { DCIS } \\
\%\end{array}$} & \multicolumn{8}{|c|}{ Invasive } \\
\hline & & & & & & \multicolumn{2}{|l|}{1} & \multicolumn{2}{|l|}{ II } & \multicolumn{2}{|l|}{ III } & \multicolumn{2}{|l|}{ IV } \\
\hline & & $\mathrm{N}$ & $\%$ & $\begin{array}{l}\text { Sample } \\
\text { number }\end{array}$ & & $\begin{array}{l}\text { Sample } \\
\text { number }\end{array}$ & $\%$ & $\begin{array}{l}\text { Sample } \\
\text { number }\end{array}$ & $\%$ & $\begin{array}{l}\text { Sample } \\
\text { number }\end{array}$ & $\%$ & $\begin{array}{l}\text { Sample } \\
\text { number }\end{array}$ & $\%$ \\
\hline \multirow[t]{3}{*}{$A B C B 1$} & Hypomethylated & 0 & 0 & 0 & & 0 & & 0 & & 0 & & 0 & \\
\hline & Normal like & 6 & 100 & 15 & 57.7 & 33 & 52.4 & 6 & 30 & 31 & 36 & 6 & 30 \\
\hline & Hypermethylated & 0 & & 11 & 42.3 & 30 & 47.6 & 14 & 70 & 57 & 64 & 14 & 70 \\
\hline \multirow[t]{3}{*}{$B R C A 1$} & Hypomethylated & 0 & 0 & 0 & 0 & 2 & 3 & 2 & 10 & 10 & 12.8 & 1 & 5.9 \\
\hline & Normal like & 6 & 100 & 26 & 100 & 63 & 97 & 18 & 90 & 68 & 87.2 & 16 & 94.1 \\
\hline & Hypermethylated & 0 & & 0 & 0 & 0 & & 0 & & 0 & & 0 & \\
\hline \multirow[t]{3}{*}{$C D K N 2 A$} & Hypomethylated & 0 & 0 & 0 & 0 & 0 & & 0 & & 0 & & 0 & \\
\hline & Normal like & 6 & 100 & 26 & 100 & 60 & 92.3 & 18 & 90 & 61 & 67.8 & 14 & 70 \\
\hline & Hypermethylated & 0 & & 0 & & 5 & 7.7 & 2 & 10 & 29 & 32.2 & 6 & 30 \\
\hline \multirow[t]{3}{*}{ ESR1 } & Hypomethylated & 0 & 0 & 0 & 0 & 0 & & 0 & & 0 & & 0 & 0 \\
\hline & Normal like & 6 & 100 & 26 & 100 & 63 & 97 & 19 & 95 & 63 & 98.4 & 19 & 100 \\
\hline & Hypermethylated & 0 & & 0 & & 2 & 3 & 1 & 5 & 1 & 1.6 & 0 & \\
\hline \multirow[t]{3}{*}{ FOXC1 } & Hypomethylated & 0 & 0 & 0 & & 0 & & 0 & & 0 & & 0 & \\
\hline & Normal like & 6 & 100 & 6 & 23 & 34 & 52.3 & 7 & 35 & 40 & 46.5 & 10 & 47.6 \\
\hline & Hypermethylated & 0 & & 20 & 77 & 31 & 47.7 & 13 & 65 & 46 & 53.5 & 11 & 52.4 \\
\hline \multirow[t]{3}{*}{ GSTP1 } & Hypomethylated & 0 & 0 & 1 & 3.8 & 0 & & 0 & & 0 & & 0 & \\
\hline & Normal like & 6 & 100 & 10 & 38.5 & 27 & 41.5 & 5 & 25 & 29 & 33 & 5 & 25 \\
\hline & Hypermethylated & 0 & & 15 & 57.7 & 38 & 58.5 & 15 & 75 & 59 & 67 & 15 & 75 \\
\hline \multirow[t]{3}{*}{ IGF2 } & Hypomethylated & 0 & 0 & 11 & 42.3 & 25 & 38.4 & 6 & 30 & 34 & 39.1 & 9 & 47.4 \\
\hline & Normal like & 6 & 100 & 14 & 53.8 & 32 & 49.2 & 9 & 45 & 32 & 36.8 & 5 & 26.3 \\
\hline & Hypermethylated & 0 & 0 & 1 & 3.9 & 8 & 12.4 & 5 & 25 & 21 & 24.1 & 5 & 26.3 \\
\hline \multirow[t]{3}{*}{ MGMT } & Hypomethylated & 0 & 0 & 0 & & 13 & 20.3 & 13 & 65 & 16 & 24.2 & 8 & 44.4 \\
\hline & Normal like & 6 & 100 & 25 & 96.1 & 50 & 78.1 & 5 & 25 & 35 & 53.1 & 5 & 27.7 \\
\hline & Hypermethylated & 0 & & 1 & 3.9 & 1 & 11.6 & 2 & 10 & 15 & 22.7 & 5 & 27.7 \\
\hline \multirow[t]{3}{*}{ MLH1 } & Hypomethylated & 0 & 0 & 4 & 15.4 & 12 & 18.5 & 6 & 32 & 4 & 4.5 & 3 & 17.6 \\
\hline & Normal like & 6 & 100 & 21 & 80.8 & 47 & 72.3 & 10 & 53 & 68 & 77.3 & 13 & 76.5 \\
\hline & Hypermethylated & 0 & & 1 & 3.8 & 6 & 9.2 & 3 & 16 & 16 & 18.2 & 1 & 5.9 \\
\hline \multirow[t]{3}{*}{ PPP2R2B } & Hypomethylated & 0 & 0 & 0 & & 0 & & 0 & & 0 & & 0 & \\
\hline & Normal like & 6 & 100 & 7 & 26.9 & 15 & 24.6 & 6 & 35 & 40 & 30.8 & 3 & 16.7 \\
\hline & Hypermethylated & 0 & & 19 & 73.1 & 46 & 75.4 & 11 & 65 & 45 & 69.2 & 15 & 83.3 \\
\hline \multirow[t]{3}{*}{ PTEN } & Hypomethylated & 0 & 0 & 0 & & 0 & & 0 & & 0 & & 0 & \\
\hline & Normal like & 6 & 100 & 21 & 80.8 & 56 & 86.2 & 13 & 65 & 23 & 27.7 & 9 & 45 \\
\hline & Hypermethylated & 0 & & 5 & 19.2 & 9 & 13.8 & 7 & 35 & 60 & 72.3 & 11 & 55 \\
\hline \multirow[t]{3}{*}{ RASSF1A } & Hypomethylated & 0 & 0 & 2 & 7.7 & 2 & 3.1 & 1 & & 0 & & 0 & \\
\hline & Normal like & 6 & 100 & 2 & 7.7 & 10 & 15.4 & 2 & 11 & 3 & 4.8 & 1 & 5.6 \\
\hline & Hypermethylated & 0 & & 22 & 84.6 & 53 & 81.5 & 17 & 90 & 60 & 95.2 & 17 & 94.4 \\
\hline
\end{tabular}

Samples were considered as hypermethylated if the\% DNA methylation was higher than the sum of two times the standard deviation and mean of the normal samples and hypomethylated if\% DNA methylation was lower than two times the standard deviation - mean of the normal samples.

value of methylation levels)/SD of methylation levels. Then the sum for the 12 genes was calculated giving one single value (Z-score) for each sample. The false discovery rate was not considered in this study due to small number of genes which were tested. All calculations were performed using Statistical Package for Science version 18.

Univariate, Kaplan-Meier analyses and the log-rank test for each parameter for single gene was performed to 
investigate which genes and parameters affect survival. Further, multivariate, the Cox proportional hazard model was used to identify independent prognostic markers for all genes and from all clinical parameters: age, stage, tumor size and grade, lymph node status, TP53 mutation status, ER, PR status, T status. Methylation status of all genes was treated as continuous and categorical. We constructed possible model candidates using all combinations of given variables. To select the best-fitted model from all candidates, we evaluated the Akaike Information Criterion (AIC) [17]. AIC gives an evaluation for model selection, which is modified by a penalty increasing with the number of variables of the model. Analyzing all combinations of given variables we selected the model that fitted best to the data indicated by a minimal value for the AIC.

\section{Results}

Methylation analysis and correlation with clinico-pathological parameters: stage and grade

A total of 48790 epigenotypes were generated through analyses of 205 CpGs in 12 genes (ABCB1 (20 CpGs), BRCA1 (19 CpGs), CDKN2A (28 CpGs), ESR1 (21 CpGs), FOXC1 (9 CpGs), GSTP1 (21 CpGs), IGF2 (17 CpGs), MGMT (9 CpGs), MLH1 (16 CpGs), PPP2R2B (14 CpGs), PTEN (19 CpGs) and RASSF1A (12 CpGs)). Six normal samples were used to estimate the normal-like methylation levels for all analyzed genes. Our analysis showed that five genes $A B C B 1, F O X C 1, G S T P 1, P P P 2 R 2 B$ and RASSF1A were the most frequently hypermethylated genes in all invasive samples as well as in the DCIS samples. PTEN was hypermethylated in invasive cancer of stage II, III and IV and MGMT was hypomethylated in invasive tumors of stage II, III and IV. CDKN2A had a normal-like methylation level in a high percentage of the DCIS samples and early stage tumors. In late stage tumors $C D K N 2 A$ showed higher percentage of hypermethylated samples compared to the early stage tumors and the DCIS. The methylation levels of ESR1 and MLH1 were normal-like both in the DCIS and the invasive tumors and BRCA1 had a normallike methylation level in almost all the DCIS and all invasive tumors (Table 3.).

Significant differences in methylation levels between the DCIS and invasive stage II tumors were observed for six genes RASSF1A, CDKN2A, MGMT, ABCB1, GSTP1 and FOXC1 $(\mathrm{p}=0.008, \mathrm{p}=0.005, \mathrm{p}=0.003, \mathrm{p}=0.006, \mathrm{p}=0.010$, $\mathrm{p}=0.010$ respectively). RASSF $1 A, A B C B 1$ and GSTP1 showed significantly higher quantitative methylation levels in late stage compared to the early stage breast carcinoma. The most significant differences in methylation levels for these three genes were between stage I and III ( $p=0.001$, $\mathrm{p}=0.022, \mathrm{p}=0.019$ respectively) and between stage I and IV $(\mathrm{p}=3.3 \mathrm{e}-6, \mathrm{p}=0.030$ and $\mathrm{p}=0.014$ respectively). PTEN and $C D K N 2 A$ methylation levels were low and increased in late stage III and IV ( $p=0.003, p=0.004$ between stage I and III, $\mathrm{p}=0.018$ and $\mathrm{p}=0.003$ between stage I and IV), while MGMT methylation levels were low and appear to decrease with tumor stage $(\mathrm{p}=4.5 \mathrm{e}-4$ between stage I and IV) (Figure 1). After correction for multiple testing (Bonferroni correction), differences in methylation levels for RASSF1A between stage I and III and stage I and IV remained significant. Absolute differences in mean methylation levels for RASSF1A were higher than 10\%. For PTEN, CDKN2A, MGMT methylation levels between stage I and

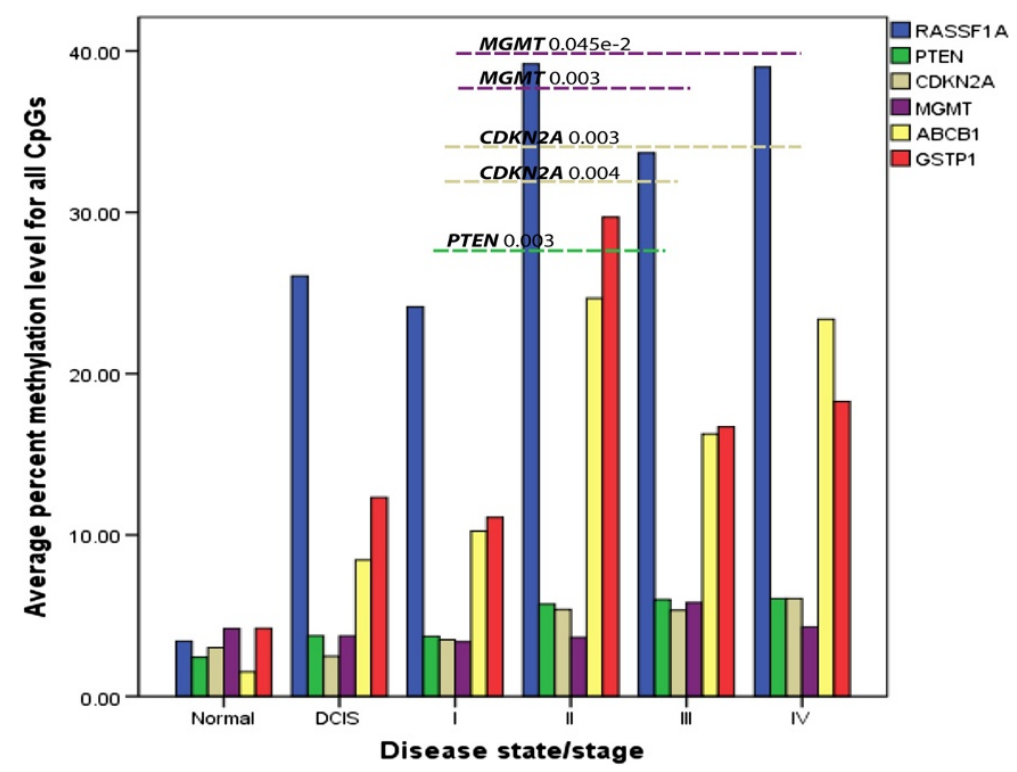

Figure 1 Average percentage of DNA methylation levels for all CpGs between normal, DCIS and invasive tumor samples (stage I, II, III and IV). 
III and stage I and IV remained significant after correction. Differences in methylation levels between the DCIS and invasive stage II tumors for MGMT also reached statistical significance after correction. Absolute differences in mean methylation levels between different stages for PTEN, CDKN2A, MGMT were less than 3\% even though they remained significant after Bonferroni correction. We next combined all the methylation data into a single variable, the methylation index, to investigate further, how methylation levels change during progression of breast cancer. At the same time we wanted to investigate the presence of general pattern which might be more robust than single genes. As shown in Figure 2, we observed significantly lower methylation levels in DCIS and stage I samples compared to stage II, III and IV samples $(p=3 e-7)$. Significant differences in methylation levels were observed between the normal breast tissue and stage II, III and IV tumors ( $\mathrm{p}=0.001, \mathrm{p}=0.006, \mathrm{p}=0.009$ respectively). Normal breast tissue showed lower levels of methylation compared with tumors mentioned above. There was no significant difference observed between the normal samples and the DCIS and stage I tumors. Also

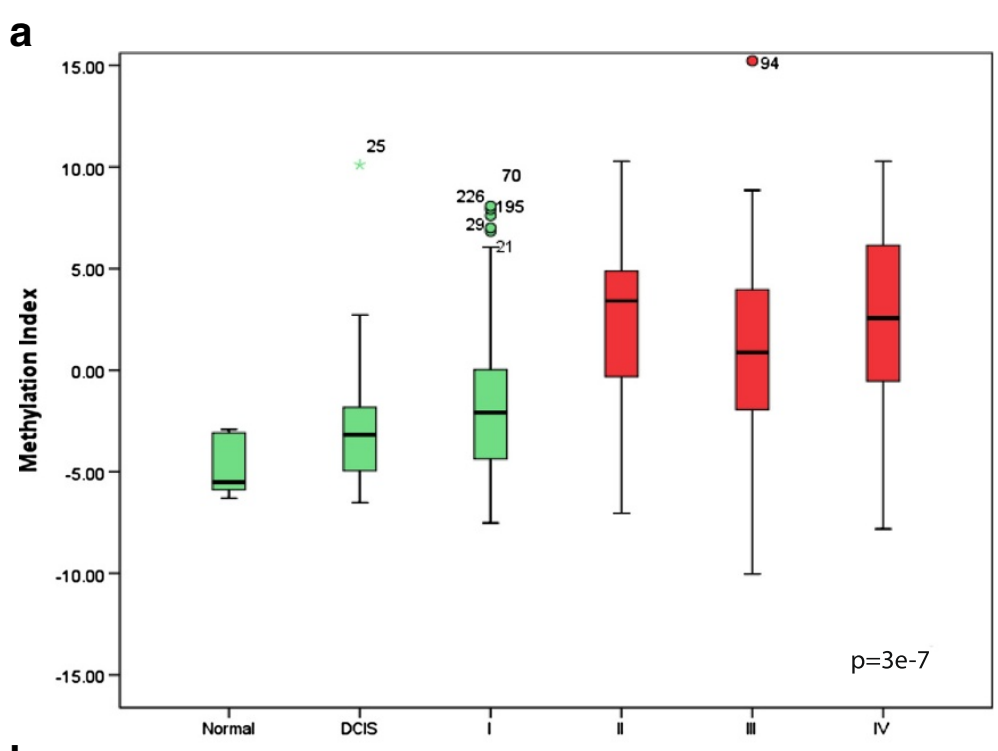

b

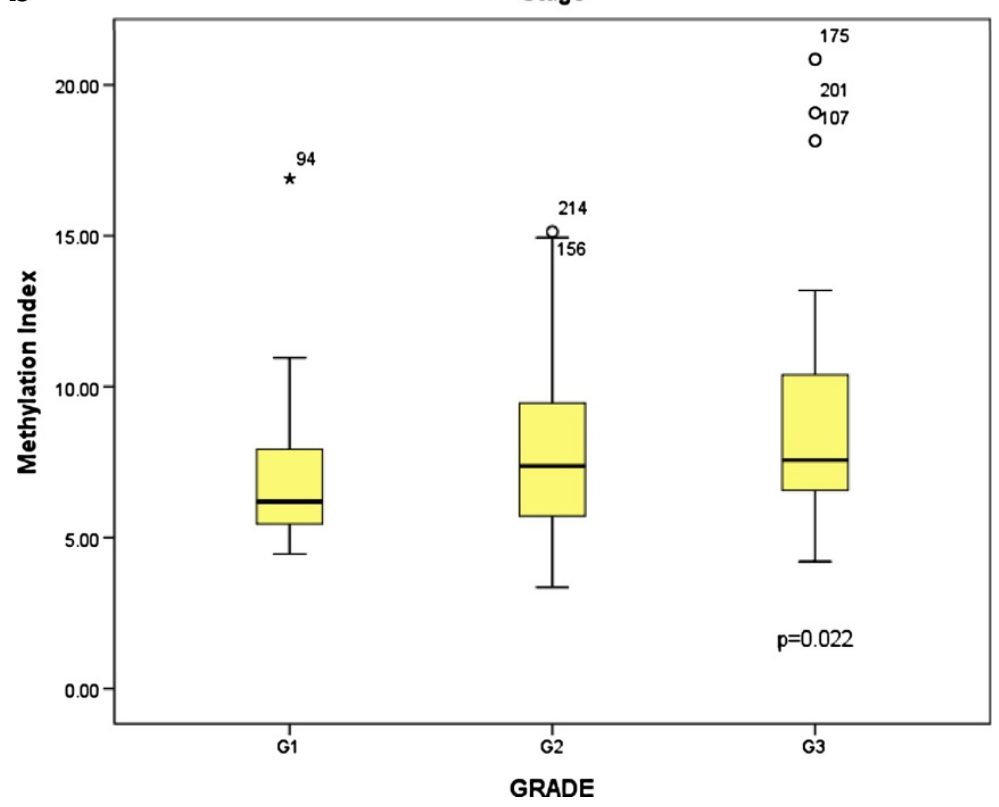

Figure 2 Boxplots illustrating significant association between methylation status and tumor stage and grade. (a) Significantly lower methylation levels in DCIS and stage I samples compared to stage II, III and IV samples was observed. (b) Also grade 1 tumors had significantly lower methylation index compared with grade 2 and grade 3 tumors. 
grade 2 and 3 tumors had significantly higher Z-scores than grade 1 tumors $(p=0.022)$. Methylation index analysis showed that Luminal A and Luminal B tumors had significantly higher Z-scores than Basal-like tumors $(\mathrm{p}=0.007)$.

\section{Correlation with TP53 mutations and hormone receptor status}

We compared the DNA methylation profiles with the TP53 mutations status and found that tumors with TP53 mutations had significantly lower DNA methylation levels then tumors with TP53 wild type in RASSF1A, PTEN, PPP2R2B, FOXC1, ABCB1 and BRCA1 ( $\mathrm{p}=0.028, \mathrm{p}=$ $0.031, \mathrm{p}=0.002, \mathrm{p}=0.017, \mathrm{p}=0.010, \mathrm{p}=0.001$ respectively). After Bonferroni correction DNA methylation levels in $P P P 2 R 2 B$ and $B R C A 1$ were still significantly lower in tumors with TP53 mutations. Significant associations with (ER) estrogen receptor status were observed for RASSF1A, PPP2R2B, GSTP1 and FOXC1 methylation levels $(\mathrm{p}=0.004, \mathrm{p}=0.012, \mathrm{p}=0.012, \mathrm{p}=0.032$, respectively). HER2 receptor status was associated with $R A S S F 1 A$, PTEN, MGMT, CDKN2A and ESR1 ( $\mathrm{p}=0.023, \mathrm{p}=3.6 \mathrm{e}-7$, $\mathrm{p}=1.1 \mathrm{e}-8, \mathrm{p}=5.8 \mathrm{e}-9, \mathrm{p}=0.017)$ and after Bonferroni correction PTEN, MGMT and CDKN2A remained significant. No significant association with (PR) progesterone receptor status was observed (Table 4). Mann-Whitney test revealed that ER and HER2 negative tumors had lower methylation levels compared with ER and HER2 positive tumors for all studied genes. Interestingly HER2 negative tumors had higher methylation levels of ESR1 compared with HER2 positive tumors $(\mathrm{p}=0.017)$. Z-score analysis showed that TP53 mutated tumors had significantly lower overall methylation levels compared to tumors with wild type TP53. Also HER2 positive tumors

\begin{tabular}{|c|c|c|c|c|c|}
\hline \multirow[t]{2}{*}{ GENE } & \multicolumn{3}{|c|}{ TP53 wt/mut $p$-value } & \multirow{2}{*}{$\begin{array}{l}\text { ER pos/neg } \\
\text { p-value }\end{array}$} & \multirow{2}{*}{$\begin{array}{l}\text { HER2 pos/neg } \\
\text { p-value }\end{array}$} \\
\hline & $\begin{array}{l}\text { WT\% } \\
\text { meth }\end{array}$ & $\begin{array}{l}\text { Mut\% } \\
\text { meth }\end{array}$ & $\overline{p \text {-value }}$ & & \\
\hline BRCA1 & 87.34 & 79.99 & 0.001 & & \\
\hline PTEN & 5.34 & 4.67 & 0.031 & & $3.6 e-6$ \\
\hline$P P P 2 R 2 B$ & 11.33 & 9.47 & 0.002 & 0.012 & \\
\hline FOXC1 & 17.12 & 13.56 & 0.017 & 0.032 & \\
\hline$A B C B 1$ & 14.52 & 10.28 & 0.010 & & \\
\hline RASSFIA & 32.48 & 26.58 & 0.028 & 0.004 & 0.023 \\
\hline GSTP1 & & & & 0.012 & \\
\hline CDKN2A & & & & & $5.8 e-9$ \\
\hline MGMT & & & & & $1.1 \mathrm{e}-7$ \\
\hline ESR1 & & & & & 0.017 \\
\hline
\end{tabular}

Indicated are \% methylation within the studied subgroups together with $\mathrm{p}$-values for differences in DNA methylation levels between genes in connection with ER, HER2 and TP53 mutation status. Mann-Whitney test. had significantly higher z-scores of methylation compared to the HER2 negative tumors (Figure 3). There was no significant association with PR and ER status.

\section{Survival analysis}

To investigate which parameters contribute to differences in survival we applied: 1) univariate analysis using KaplanMeier modeling and the log-rank test for each gene and each clinical parameter and 2) multivariate analysis using Cox hazard proportional model to all variables. To select the best-fitted model from all model candidates, we evaluated the Akaike Information Criterion [17].

Univariate survival analysis identified methylation status of $P P P 2 R 2 B$ as significant predictor of overall survival. As expected, grade, estrogen receptor status, TP53 status and stage also appeared as significant predictors of survival. The Kaplan-Meier plot (Figure 4) showed a significant difference in survival between hypermethylated and normal-like samples for $P P P 2 R 2 B(\mathrm{p}=0.012)$ indicating that patients with hypermethylated genes had better survival. Breast cancer specific survival was significantly improved in patients with hyper-methylated promoters for $P P P 2 R 2 B(\mathrm{p}=0.012)$.

Further, multivariate survival analysis was performed in order to investigate if any of the methylation markers were independent prognostic markers, using both, categorical and continuous methylation data. We constructed possible model candidates using all relevant parameters and methylation data. Using calculated AIC, the best model that fits to the survival data was assessed. For categorical methylation data AIC identified a model explaining survival, which included the methylation status of $I G F 2$, GSTP1, estrogen receptor status, TP53, N status and stage. The estimated coefficients, the hazard ratio, the p-values, and the $95 \%$ confidence intervals of the hazard ratio were summarized in Table 5. For continuous methylation data the best model explaining survival included TP53, T status, $\mathrm{N}$ status, estrogen receptor status and methylation status of IGF2 and GSTP1. The estimated coefficients, the hazard ratio, the p-values, and the $95 \%$ confidence intervals of the hazard ratio were summarized in Table 6. For both, categorical and continuous methylation data IGF2 and GSTP1 $(\mathrm{p}=0.009$ and $\mathrm{p}=0.014)$ were significant together with ER status $(\mathrm{p}=0.008)$, TP53 status $(\mathrm{p}=6.6 \mathrm{e}-5)$ and $\mathrm{N}$ status $(\mathrm{p}=0.047)$. For these models, we also described statistical significance for the likelihood ratio, Wald and log-rank tests in the Tables 5 and 6.

\section{Discussion}

The aim of this study was to quantitatively determine the methylation levels in the promoter region of 12 genes in breast cancer patients of all stages and to investigate stage specific changes in tumors. In addition, we wanted to evaluate the association between clinico-pathological 

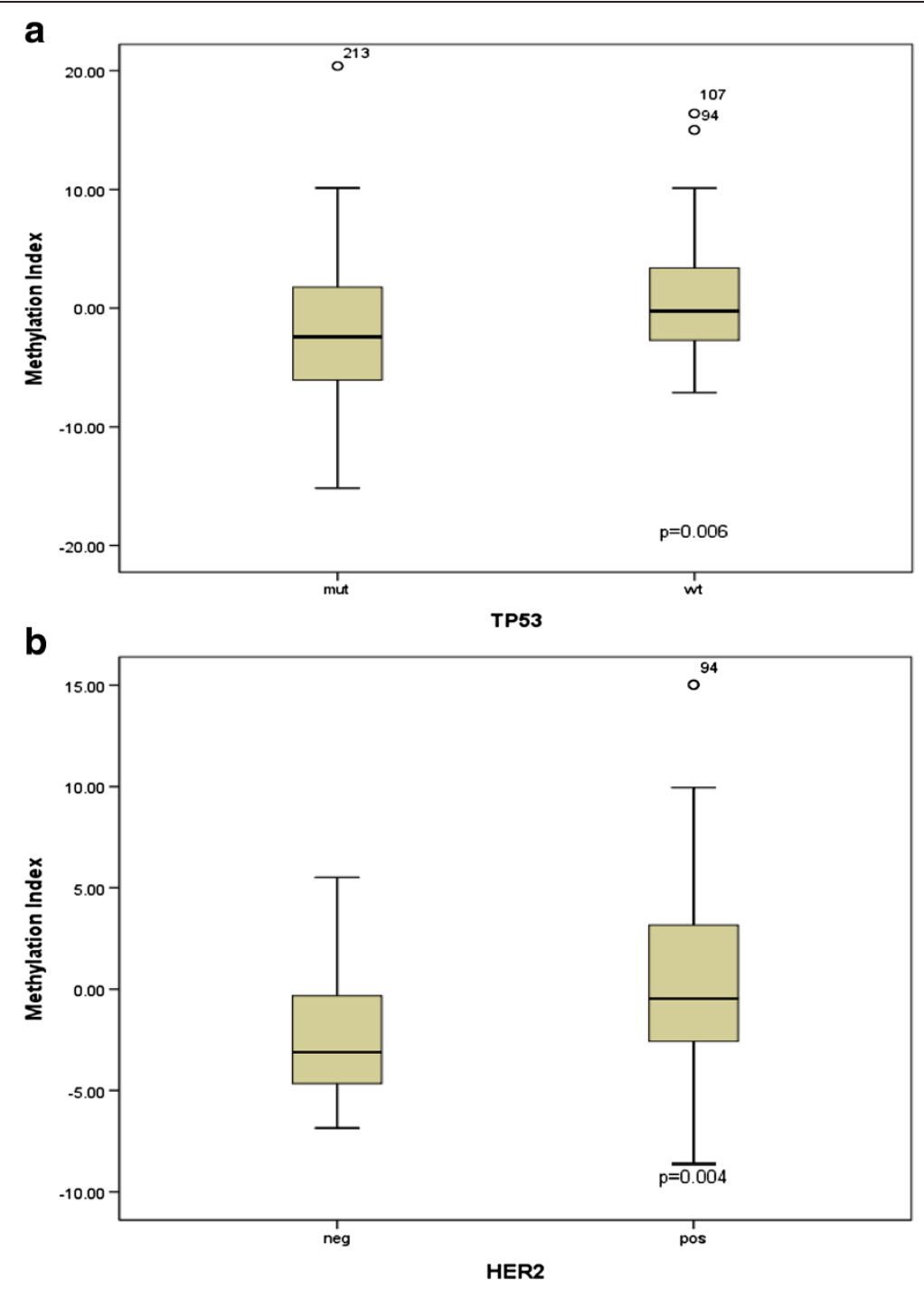

Figure 3 Boxplots illustrating significant association between methylation status and HER2 and TP53 status. (a) TP53 mutated tumors had significantly lower overall methylation levels compared to tumors with wild type TP53. (b) HER2 positive tumors had significantly higher methylation index compared to the HER2 negative tumors.

factors (ER, HER2 status, grade) including survival and methylation levels of these genes.

In the present study, five genes, $A B C B 1, F O X C 1$, GSTP1, PPP2R $2 B$ and RASSF1A were hypermethylated already in early stage breast cancer (stage I and II). For all five genes hypermethylation was also detected in DCIS suggesting that inactivation of these genes is a frequent event in the process of mammary tumorigenesis. We found that RASSF1A was hypermethylated in approximately $85 \%$ of all invasive tumors and DCIS, and our results are in agreement with such a high incidence of RASSF1A methylation [18-20]. RASSF1A is a putative tumor-suppressor gene. It belongs to an increasing list of tumor suppressor genes that are frequently inactivated by promoter methylation rather than by somatic mutations [21]. Since we detected a constant hypermethylation of RASSF1A in all of the different stages of the breast carcinomas we can suggest that hypermethylation of RASSF1A is an early event during breast cancer pathogenesis and also the main mechanism of inactivation. In our study, GSTP1 was found to be hypermethylated in different stages of breast carcinomas, for early stages (I and II) our results are in agreement with previous reports [22,23]. The frequency of GSTP1 promoter hypermethylation in stage III and IV (around 70\%) was found to be higher than reported previously [23,24]. It is known that GSTP1 plays a role in detoxification of potential carcinogens and that loss of the expression of GSTP1 will lead to DNA damage of breast cells and they will be more easily exposed to carcinogens [25]. Loss of GSTP1 expression and its 


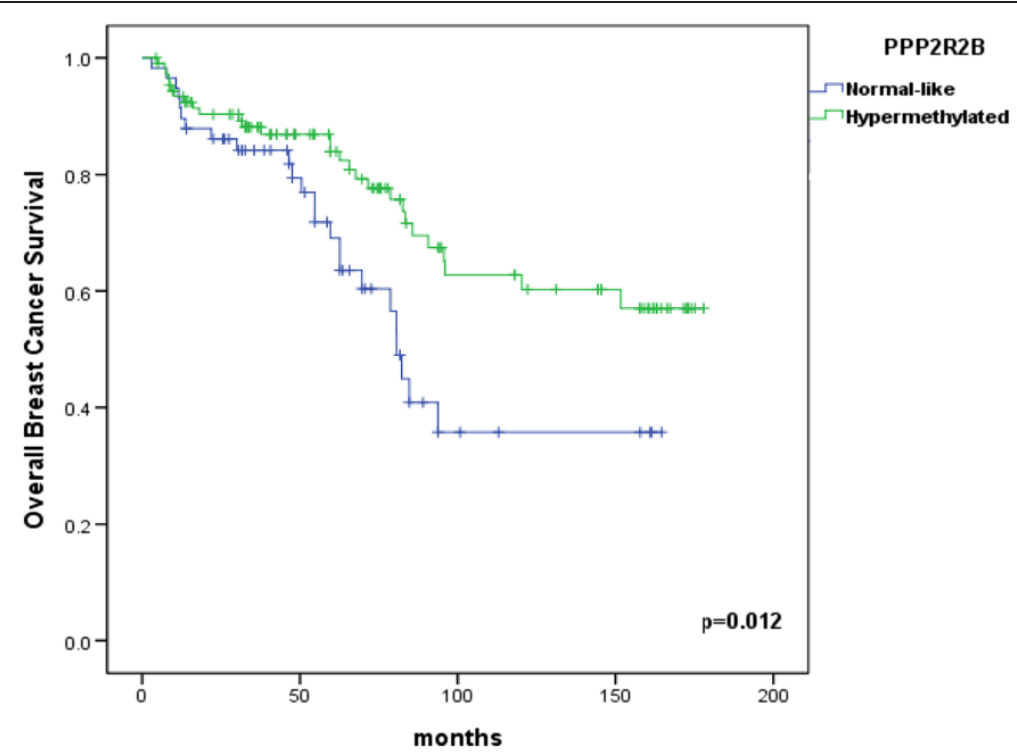

Figure 4 Kaplan-Meier plots of overall survival for patients with normal-like or hyper methylated PPP2R2B promoter. The $p$ value waS calculated using a long-rank test.

potential role in breast carcinogenesis was observed in high proportion of breast tumors [23]. It appears that promoter hypermethylation is associated with loss of GSTP1 expression [26]. Important question is when the promoter hypermethylation of the GSTP1 gene starts to play a role in tumor progression? We found that already in DCIS there is a high proportion of hypermethylated GSTP1 (58\%), which indicates that GSTP1 promoter hypermethylation is an early event in breast carcinogenesis. From our analysis we observed that hypermethylation of $A B C B 1$, $P P P 2 R 2 B$ and FOXC1 is also an early event in breast carcinogenesis since our results indicate high level of hypermethylation of these genes already in DCIS which was reported before [4]. We found frequencies of methylation for CDKN2A MGMT and MLH1 similar to previously published reports [27,28]. According to our results, we could suggest that hypermethylation of $C D K N 2 A$ is possible event leading to its inactivation in late stage breast cancers. PTEN is a tumor suppressor gene. Its product PTEN protein works as a negative regulator of the Akt pathway, leading to suppression of apoptosis and increasing cell survival [29]. We suggest here that epigenetic silencing of PTEN might be an early event in initiation of cancer and also the mechanism of its inactivation. Next, combining all the methylation data into a single variable, the methylation index, we investigate how methylation levels change during progression of breast cancer. Our analysis showed that the methylation pattern of all genes included in this study is changing during breast cancer progression. DCIS and stage I tumors had similar

Table 5 Multivariate survival analysis - categorical methylation data

\begin{tabular}{|c|c|c|c|c|c|}
\hline Covariate & Baseline & Coefficient $\left(b_{i}\right)$ & $H R\left(\exp \left(b_{i}\right)\right.$ & $\mathrm{p}$-value & $95,0 \% \mathrm{Cl}$ for $\operatorname{Exp}(\mathrm{B})$ \\
\hline TP53 & TP53 wild type & -2.332 & 0.097 & $6.6 e-5$ & $(0.030-0.305)$ \\
\hline ER & ER positive & 1.481 & 4.401 & 0.008 & $(1.460-13.269)$ \\
\hline Stage II & Stage I & 2.260 & 9.584 & 0.013 & $(1.593-57.667)$ \\
\hline Stage III & Stage I & -0.011 & 0.988 & 0.992 & $(0.088-11.019)$ \\
\hline N1 & No & -6.362 & 0.529 & 0.338 & $(0.143-1.947)$ \\
\hline N2 & No & 1.673 & 5.332 & 0.047 & $(1.019-27.885)$ \\
\hline IGF2 & Normal like IGF2 & 1.597 & 4.940 & 0.009 & $(1.472-16.581)$ \\
\hline GSTP1 & Normal like GSTP1 & -1.621 & 0.197 & 0.014 & $(0.053-0.729)$ \\
\hline$P P P 2 R 2 B$ & Normal like $P P P 2 R 2 B$ & -0.972 & 0.378 & 0.085 & $(0.125-1.143)$ \\
\hline CDKN2A & Normal like CDKN2A & -0.839 & 0.432 & 0.133 & $(0.144-1.294)$ \\
\hline
\end{tabular}

The variables that the minimum AIC selected. ( $p$-values: 3.4e-7 for likelihood ratio test, 3.4e-4 for Wald test, 3.2e-6 for log-rank test). Positive hazard ratios indicate an increased risk of dying from breast cancer and are calculated for the different covariates. 
Table 6 Multivariate survival analysis - continuous methylation data

\begin{tabular}{|c|c|c|c|c|c|}
\hline Covariate & Baseline & Coefficient $\left(b_{i}\right)$ & $H R\left(\exp \left(b_{i}\right)\right.$ & $p$-value & $95,0 \% \mathrm{Cl}$ for $\operatorname{Exp}(\mathrm{B})$ \\
\hline GSTP1 & Normal like GSTP1 & -0.066 & 0.935 & $4 e-4$ & $(0.902-0.970)$ \\
\hline IGF2 & Normal like IGF2 & 0.079 & 1.082 & 0.002 & $(1.031-1.136)$ \\
\hline MLHI & Normal like MLH1 & -0.552 & 0.575 & 0.135 & $(0.278-1.187)$ \\
\hline ER & ER positive & 1.659 & 5.256 & 0.002 & $(1.804-15.317)$ \\
\hline TP53 & TP53 wild type & -2.169 & 0.114 & 0.002 & $(0.028-0.450)$ \\
\hline N 1 & No & 1.684 & 5.391 & 0.022 & $(1.265-22.975)$ \\
\hline N 2 & NO & 1.706 & 5.511 & 0.032 & $(1.151-26.371)$ \\
\hline T2 & $\mathrm{T} 1$ & 1.937 & 6.942 & 0.035 & $(1.143-42.157)$ \\
\hline T3 & $\mathrm{T} 1$ & 0.050 & 1.051 & 0.960 & $(0.143-7.716)$ \\
\hline $\mathrm{T} 4$ & $\mathrm{~T} 1$ & 1.125 & 3.081 & 0.258 & $(0.437-21.692)$ \\
\hline
\end{tabular}

The variables that the minimum AIC selected. ( $p$-values: 1.2e-7 for likelihood ratio test, $5.8 \mathrm{e}-4$ for Wald test, $6.7 \mathrm{e}-6$ for log-rank test).

Positive hazard ratios indicate an increased risk of dying from breast cancer and are calculated for the different covariates.

methylation levels with no significant difference. Stage II tumors showed the most significant difference in methylation levels when compared with DCIS and stage I and then in stage III and IV methylation levels were lower but still significantly higher than in DCIS and stage I. It remains unclear how this process of dramatic change in stage II is achieved and driven further during breast cancer progression. To our knowledge only one group investigate stage dependent DNA methylation in breast cancer using DCIS and all four stages of invasive tumors [30]. They identified 33 cancer specific genes that were either highly methylated in early stage breast cancer or showed stage dependent methylation pattern, lower methylation frequency in early stage breast cancers and a higher methylation frequency in late stage breast cancers. None of our selected genes were methylated in this study. Additional studies of the relationship between DNA methylation in tumors and tumor stages are necessary.

In the present study we showed the associations between DNA methylation levels of candidate genes and the TP53 mutation status, estrogen receptor status, and HER2 status. The TP53 tumor suppressor gene has a central role in cell cycle regulation, DNA repair and apoptosis, and a large number of reports have discussed the important role of TP53 alterations in breast cancer. Also, a number of studies have shown that breast tumors with TP53 mutations are strongly associated with poor prognosis and lacking methylation in a number of regulatory genes [31,32]. Additionally, studies on different expression subtypes in breast cancer showed that different subtypes have a different underlying biology reflected in methylation and is strongly influenced by TP53 mutation status. It was shown that basal-like tumors are TP53 mutated and unmethylated $[6,33]$. In present study we have identified $26,6 \%$ of breast tumors with TP53 mutations and significantly lower levels of DNA methylation in RASSF1A, PTEN, PPP2R2B, FOXC1, ABCB1 and BRCA1 compared to tumors with wild type TP53. Since in our study we had low number of basal-like tumors (37 out of 238) we cannot confirm association between TP53 mutated and unmethylated tumors and basal-like but most of the basal-like samples were TP53 mutated and had lowest methylation levels compared to other subtypes (data not shown). The status of ER and HER2 have been recognized as important prognostic factors in patients with breast cancer, in addition to a predictive marker for the response to treatment with endocrine and trastuzumab therapy. Identification of genes with subtype-specific methylation revealed that RASSF1A and GSTP1 were highly methylated in Lum B tumors [33]. These two genes were reported previously to be significantly more methylated in ER-positive than ER-negative tumors [34]. In the present study we showed the same trend in ER-positive tumors compared to ER-negative for four genes and RASSF1A and GSTP1 were among them. The same group reported that HER2 positive tumors had higher methylation level for these two genes compared to HER2-negative tumors, which is again in accordance with our study. Furthermore, in a study on methylation in breast cancer and breast cancer molecular subtypes it was shown that RASSF1A is hypermethylated in HER2 positive breast tumors (ERBB2 and luminal B) [35]. In our study RASSF1A was hypermethylated in ERBB2 and luminal B tumors (data not shown). Taken all together, these results, suggest that methylation plays a significant role in the different breast tumor phenotypes.

We report here for the first time the $P P P 2 R 2 B$ methylation status as significant predictor for breast cancer survival as well as for overall survival. $P P P 2 R 2 B$ is a candidate tumor suppressor gene and it was shown that changes in DNA methylation of this gene contribute to its expression [36]. Further, multivariate analysis showed that IGF2 and GSTP1 were independent prognostic markers. Recently, it has been shown that the absence of GSTP1 protein expression correlate with promoter 
hypermethylation and with improved survival in invasive breast cancer samples [37]. Hypermethylation of GSTP1 is a well established biomarker for hormone dependent cancers. Our previous analyses suggest that methylation of GSTP1 in locally advanced breast cancer patients treated with doxorubicin was associated to survival [3]. However, GSTP1 had no effect on treatment response.

\section{Conclusions}

Here we report aberrant methylation levels of $A B C B 1$, FOXC1, GSTP1, PPP $2 R 2 B$ and RASSF1A in DCIS and stage I-IV providing evidence that suggests that changes in methylation level is an early event and may also be important in progression to later stages of breast cancer. We also report that methylation levels of important breast cancer genes are associated to hormone receptor status and TP53 mutation status suggesting mechanisms of deactivation of tumor suppressor genes in breast cancer. Further studies are necessary to identify which methylation gene profiles are of predictive and which of prognostic value.

\section{Additional file}

Additional file 1: PCR and pyrosequencing primers. Sequences of primers used for amplification and pyrosequencing reactions, Genbak accession numbers and nucleotides (Nt) corresponding to the amplified fragments as well as the annealing temperatures for the respective PCR amplifications. CpGs are numbered in the order of appearance from the 5 ' end of an amplification product. $Y=$ pyrimidine.

\section{Competing interests}

The authors declare that they have no competing interests.

\section{Authors' contribution}

JK performed data analyses and wrote the manuscript. ED perfomed laboratory experiments.TF, HE and HS were involved in the statistical analyses. PEL and FW were responsible for the patient cohorts and IB for the control samples. ALBD, JT and VNK initiated and designed the study and participated in writing the manuscript. All authors have read and approved the final manuscript.

\section{Acknowledgements}

The authors gratefully acknowledge the women participating in this study for their contribution. This work was supported by grant from The Norwegian Cancer Society (VNK), the Norwegian Research Council (VNK) and the FrenchNorwegian cooperation program Aurora grant (JT,VNK). JK is a fellow of the Akershus University hospital, Division of Medicine, University of Oslo. TF is a fellow of the Faculty Division the Norwegian Radium Hospital, University of Oslo. HE is a postdoctoral fellow of the Norwegian Cancer Society.

\section{Author details}

'Department of Clinical Molecular Biology and Laboratory Science (EpiGen), Akershus University hospital, Division of Medicine, 1476 Lørenskog, Norway. ${ }^{2}$ Institute for Clinical Medicine, Faculty of Medicine, University of Oslo, 0372 Oslo, Norway. ${ }^{3}$ Department of Genetics, Institute for Cancer Research, OUS Radiumhospitalet Montebello, 0310 Oslo, Norway. ${ }^{4}$ Laboratory for Epigenetics, Centre National de Génotypage, CEA - Institut de Génomique, 91000 Evry, France. ${ }^{5}$ Department of Surgery, Uppsala University Hospital, Uppsala, Sweden. ${ }^{6}$ Department of Surgery, Akerhus University Hospital, Oslo, Norway. ${ }^{7}$ Section of Oncology, Institute of Medicine, University of Bergen and Department of Oncology, Haukeland University Hospital, N5021 Bergen, Norway.
Received: 24 May 2013 Accepted: 1 October 2013

Published: 5 October 2013

\section{References}

1. Jovanovic J, Rønneberg JA, Tost J, Kristensen V: The epigenetics of breast cancer. Mol Oncol 2010, 4:242-254.

2. Jones PA, Baylin SB: The epigenomics of cancer. Cell 2007, 128:683-692.

3. Dejeux E, Rønneberg JA, Solvang H, Bukholm I, Geisler S, Aas T, Gut IG, Børresen-Dale AL, Lønning PE, Kristensen VN, Tost J: DNA methylation profiling in doxorubicin treated primary locally advanced breast tumours identifies novel genes associated with survival and treatment response. Mol Cancer 2010, 9:68.

4. Muggerud AA, Rønneberg JA, Wärnberg F, Botling J, Busato F, Jovanovic J, Solvang H, Bukholm I, Børresen-Dale AL, Kristensen VN, Sørlie T, Tost J: Frequent aberrant DNA methylation of $A B C B 1, F O X C 1, P P P 2 R 2 B$ and PTEN in ductal carcinoma in situ and early invasive breast cancer. Breast Cancer Res 2010, 12:R3.

5. Geisler S, Børresen-Dale AL, Johnsen H, Aas T, Geisler J, Akslen LA, Anker G, Lønning PE: TP53 gene mutations predict the response to neoadjuvant treatment with 5 -fluorouracil and mitomycin in locally advanced breast cancer. Clin Cancer Res 2003, 9:5582-8.

6. Rønneberg JA, Fleischer T, Solvang HK, Nordgard SH, Edvardsen H, Potapenko I, Nebdal D, Daviaud C, Gut I, Bukholm I, Naume B, Børresen-Dale AL, Tost J, Kristensen V: Methylation profiling with a panel of cancer related genes: association with estrogen receptor, TP53 mutation status and expression subtypes in sporadic breast cancer. Mol Ocol 2011, 5:61-76.

7. Tost J, Schatz P, Schuster M, Berlin K, Gut IG: Analysis and accurate quantification of $\mathrm{CpG}$ methylation by MALDI mass spectrometry. Nucleic Acids Res 2003, 31:e50.

8. David GL, Yegnasubramanian S, Kumar A, Marchi VL, De Marzo AM, Lin X, Nelson WG: MDR1 Promoter hypermethylation in MCF-7 human breast cancer cells: changes in chromatin structure induced by treatment with 5-Aza-cytidine. Cancer Biol Therap 2004, 3:540-548.

9. Esteller M, Corn PG, Baylin SB, Herman JG: A gene hypermethylation profile of human cancer. Cancer Res 2001, 61:3225-3229.

10. Lapidus RG, Nass SJ, Butash KA, Parl FF, Weitzman SA, Graff JG, Herman JG, Davidson NE: Mapping of ER gene CpG island methylation-specific polymerase chain reaction. Cancer Res 1998, 58:2515-2519.

11. Esteller M, Corn PG, Urena JM, Gabrielson E, Baylin SB, Herman JG: Inactivation of glutathione $\mathrm{S}$-transferase $\mathrm{P} 1$ gene by promoter hypermethylation in human neoplasia. Cancer Res 1998, 58:4515-4518.

12. Issa JP, Vertino PM, Boehm CD, Newsham IF, Baylin SB: Switch from monoallelic to biallelic human IGF2 promoter methylation during aging and carcinogenesis. Proc Natl Acad Sci U S A 1996, 93:11757-11762.

13. Keen JC, Garrett-Mayer E, Pettit C, Mack KM, Manning J, Herman JG, Davidson NE: Epigenetic regulation of protein phosphatase 2A (PP2A), lymphotactin (XCL1) and estrogen receptor alpha (ER) expression in human breast cancer cells. Cancer Biol Therap 2004, 3:1304-1312.

14. Garcia JM, Silva J, Pena C, Garcia V, Rodriguez R, Cruz MA, Cantos B, Provencio M, Espana P, Bonilla F: Promoter methylation of the PTEN gene is a common molecular change in breast cancer. Genes Chromosomes Cancer 2004, 41:117-124.

15. Yan PS, Shi H, Rahmatpanah F, Hsiau TH, Hsiau AH, Leu YW, Liu JC, Huang TH: Differential distribution of DNA methylation within the RASSF1A CpG island in breast cancer. Cancer Res 2003, 63:6178-6186.

16. Sorlie T, Perou CM, Tibshirani R, Aas T, Geisler S, Johnsen H, Hastie T, Eisen MB, van de Rijn M, Jeffrey SS, Thorsen T, Quist H, Matese JC, Brown PO, Botstein D, Lonning PE, Borresen-Dale AL: Gene expression patterns of breast carcinomas distinguish tumor subclasses with clinical implications. Proc Natl Acad Sci U S A 2001, 98:10869-10874.

17. Akaike H: A new look at the statistical identification model. IEEE Trans Automatic Control 1974, 19:716-723.

18. Fackler MJ, McVeigh M, Evron E, Garrett E, Mehrotra J, Polyak K, Sukumar S, Argani P: DNA methylation of RASSF1A, HIN-1, RAR- $\beta$, cyclin D2 and twist in in situ and invasive lobular breast carcinoma. Int I Cancer 2003, 107:970-975.

19. Yeo W, Wong WL, Wong N, Law BK, Tse GM, Zhong S: High frequency of promoter hypermethylation of RASSF1A in tumorous and non-tumourous tissue of breast cancer. Pathology 2005, 37:125-130. 
20. Kioulafa M, Kaklamanis L, Mavroudis D, Georgoulias V, Lianidou ES: Prognostic significance of RASSF1A promoter methylation in operable breast cancer. Clin Biochem 2009, 42:970-975.

21. Jones PA, Baylin SB: The fundamental role of epigenetic events in cancer. Nat Rev Genet 2002, 3:415-428.

22. Lee JS: GSTP1 promoter hypermethylation is an early event in breast carcinogenesis. Virchows Arch 2007, 450:637-642.

23. Arai T, Miyoshi Y, Kim SJ, Taguchi T, Tamaki Y, Noguchi S: Association of GSTP1 CpG islands hypermethylation with poor prognosis in human breast cancers. Breast Cancer Res Treat 2006, 100:169-176.

24. Hoque MO, Feng Q, Toure P, Dem A, Critchlow CW, Hawes SE, Wood T, Jeronimo C, Rosenbaum E, Stern J, Yu M, Trink B, Kiviat NB, Sidransky D: Detection of aberrant methylation of four genes in plasma DNA for the detection of breast cancer. J Clin Oncol 2006, 24:4262-4269.

25. Lee WH, Morton RA, Epstein JI, Brooks JD, Campbell PA, Bova GS, Hsieh WS, Isaacs WB, Nelson WG: Cytidine methylation of regulatory sequences near the pi-class glutathione S-transferase gene accompanies human prostatic carcinogenesis. Proc Natl Acad Sci U S A 2004, 91:11733-11737.

26. Zhong S, Tang MW, Yeo W, Liu C, Lo YM, Johnson PJ: Silencing of GSTP1 gene by $\mathrm{CpG}$ island DNA hypermethylation in HBV-associated hepatocellular carcinomas. Clin Cancer Res 2002, 8:1087-1092.

27. Nielsen NH, Roos G, Emdin SO, Landberg G: Methylation of the p16 (Ink4a) tumor suppressor gene 5'-CpG island in breast cancer. Cancer Lett 2001, 163:59-59.

28. Viswanathan M, Solomon SP, Tsuchida N, Selvam GS, Shanmugam G: Methylation of E-cadherin and hMLH1 genes in Indian sporadic breast carcinomas. Indian J Exp Biol 2006, 44:115-119.

29. Khan S, Kumagai T, Vora J, Bose N, Sehgal I, Koeffler PH, Bose S: PTEN promoter is methylated in a proportion of invasive breast cancers. Int I Cancer 2004, 112:407-410.

30. Jeschke J, Van Neste L, Glöckner SC, Dhir M, Calmon MF, Deregowski V, Van Criekinge W, Vlassenbroeck I, Koch A, Chan TA, Cope L, Hooker CM, Schuebel KE, Gabrielson E, Winterpacht A, Baylin SB, Herman JG, Ahuja N: Biomarkers for detection and prognosis of breast cancer identified by a functional hypermethylome screen. Epigenetics 2012, 7:701-709.

31. Feki A, Irminger-Finger I: Mutational spectrum of $p 53$ mutations in primary breast and ovarian tumors. Crit Rev Oncol Hematol 2004, 52:103-116.

32. Feng W, Shen L, Wen S, Rosen DG, Jelinek J, Hu X, Huan S, Huang M, Liu J, Sahin AA, Hunt KK, Bast RC Jr, Shen Y, Issa JP, Yu Y: Correlation between CpG methylation profiles and hormone receptor status in breast cancers. Breast Cancer Res 2007, 9:R57.

33. Holm K, Hegardt C, Staaf J, Vallon-Christersson J, Jönsson G, Olsson H, Borg A, Ringnér M: Molecular subtypes of breast cancer are associated with characteristic DNA methylation patterns. Breast Cancer Res 2010, 12:R36.

34. Eiji S, Masaru S, Myung-Shin S, Nguyen SL, Anh-Thu V, Giuliano AE, Dave SB $\mathrm{H}$ : Estrogen receptor and HER2/neu status affect epigenetic differences of tumor-related genes in primary breast tumors. Breast Cancer Res 2008, 10:R46.

35. Bediaga NG, Acha-Sagredo A, Guerra I, Viguri A, Albaina C, Ruiz Diaz I, Rezola R, Alberdi MJ, Dopazo J, Montaner D, Renobales M, Fernández AF, Field JK, Fraga MF, Liloglou T, de Pancorbo MM: DNA methylation epigenotypes in breast cancer molecular subtypes. Breast Cancer Res 2010, 12:R77.

36. Keen JC, Garrett-Mayer E, Pettit C, Mack KM, Manning J, Herman JG, Davidson NE: Epigenetic regulation of protein phosphatase 2A (PP2A), lymphotactin (XCL1) and estrogen receptor alpha (ER) expression in human breast cancer cells. Cancer Biol Ther 2004, 3:1304-1312.

37. Huang J, Tan PH, Thiyagarajan J, Bay BH: Prognostic significance of glutathione S-transferase-pi in invasive breast cancer. Mod Pathol 2003, 16:558-565.

doi:10.1186/1471-2407-13-456

Cite this article as: Klajic et al:: Quantitative DNA methylation analyses reveal stage dependent DNA methylation and association to clinicopathological factors in breast tumors. BMC Cancer 2013 13:456.

\section{Submit your next manuscript to BioMed Central and take full advantage of:}

- Convenient online submission

- Thorough peer review

- No space constraints or color figure charges

- Immediate publication on acceptance

- Inclusion in PubMed, CAS, Scopus and Google Scholar

- Research which is freely available for redistribution

Submit your manuscript at www.biomedcentral.com/submit
() Biomed Central 